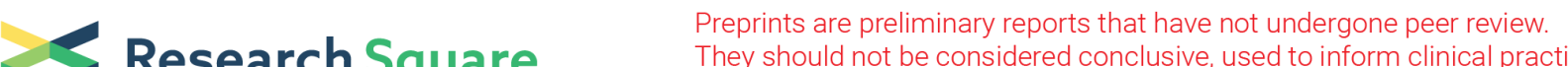 $\begin{array}{ll}\text { Research Square } & \text { They should not be considered conclusive, used to inform clinical practice, } \\ \text { or referenced by the media as validated information. }\end{array}$
}

\section{Molecular Epidemiology of Carbapenem-Resistant Klebsiella pneumoniae Infections in Southwest China}

\section{Xiaopin $\mathrm{Hu}$}

Medical School of Guizhou University

\section{Guohang Yuan}

Guizhou Provincial People's Hospital

\section{Yaoyao Wu}

Guizhou Provincial People's Hospital

Weijia Liu

Guizhou Provincial People's Hospital

Xiangyan Zhang

Guizhou Provincial People's Hospital

Lin Liu ( $\square$ liulin@gzu.edu.cn )

Guizhou Provincial People's Hospital https://orcid.org/0000-0002-1947-0809

\section{Research}

Keywords: carbapenem-resistant Klebsiella pneumoniae, clinical characteristics, microbiological characteristics, resistance mechanism

Posted Date: June 12th, 2020

DOl: https://doi.org/10.21203/rs.3.rs-34601/v1

License: (c) (1) This work is licensed under a Creative Commons Attribution 4.0 International License. Read Full License 


\section{Abstract}

Background: We determined epidemiological characteristics and resistance mechanisms of carbapenemresistant Klebsiella pneumoniae (CRKP) strains found in Southwest China and assessed disease burden to provide evidence-based strategies for control and treatment of CRKP infection.

Methods: A total of 159 strains of CRKP were isolated from sputa, blood, urine, ascites and wound secretions from three tertiary hospitals in Southwest China between August $1^{\text {st }}, 2018$ and December $31^{\text {st }}$, 2019. The sensitivity of each strain to 12 antibiotic agents was determined by micro-broth dilution. Identification of carbapenemase genes and multi-locus sequence typing (MLST) were performed using polymerase chain reaction (PCR). The disease burdens of patients with CRKP were assessed based on invasive procedures, antibiotic use, laboratory tests and clinical outcomes.

Results: Of 159 CRKP strains analyzed, $50.9 \%$ were isolated from sputum samples. The percentage of patients who underwent invasive procedures before positive cultures for CRKP were detected was $96.3 \%$. The mortality of blood infection was highest (66.6\%) among patients with CRKP infection. All strains were insensitive to carbapenems. The resistance rates to levofloxacin and amikacin were $85.5 \%$ and $81.8 \%$, respectively. All CRKP strains produced carbapenemases, with a majority of isolates (81.1\%) producing KPC-2. The MICs of strains harbouring both KPC-2 and NDM-1 were higher than those of strains with only KPC-2 or NDM-1. ST11 is the most popular clonotype found in Southwest China.

Conclusions: CRKP strains in Southwest China are characterized by strong drug resistance and associated with poor clinical prognoses. It is therefore urgent to both strengthen control measures and improve prevention awareness.

\section{Background}

Carbapenem-resistant Enterobacteriaceae (CRE) have recently emerged as a class of bacterial pathogens that pose a significant threat, both to global public health and to high-risk patients undergoing lifethreatening procedures[1]. Although multiple genera of Enterobacteriaceae have been found to carry carbapenemase enzymes, carbapenem-resistant Klebsiella pneumoniae (CRKP)remains the most epidemiologically important on a global scale [2]. The prevalence of healthcare-related CRE infections has increased from $1.2 \%$ in 2001 to $4.2 \%$ in 2011 according to the National Healthcare Safety Network (NHSN). Similarly, the National Nosocomial Infection Surveillance System (NNIS), which concentrated mainly on Klebsiella species, reported an increase in infection rate from 1.6-10.4\% [3]. Clinical studies have revealed high failure rates associated with treatment of CRKP infections, and infection mortality rates range from 22-72\% [4, 5]. Extensive resistance phenotypes have resulted in a dearth of clinical therapeutic choices for treating nosocomial infections due to CRKP, which has, in turn, posed additional difficulties for patient management. Since the initial identification of a Klebsiella pneumoniae carbapenemase (KPC)-producing K. pneumoniae isolate in 2007, CRKP has been confirmed in almost every province in China [6, 7]. A multicenter study that collected 999 strains of CRE from 27 provinces and 
regions across China between 2014 and 2015 found that CRKP accounted for the vast majority of strains (70\%), far higher than both Escherichia coli (16\%) and other Enterobacteriaceae (13\%) [7]. There are a few reports regarding the distribution and resistance rate of CRKP in China [7-10]. However, there remains a lack of information concerning the molecular epidemiology of CRKP in Southwest China. In this study, CRKP strains collected from three tertiary hospitals in Southwest China were analyzed. The clinical characteristics, laboratory tests and outcomes of patients with CRKP infection were also compared. As a whole, this study provides critical information to inform the treatment and prevention of CRKP infections.

\section{Methods}

\section{Strain collection and identification}

A total of 159 CRKP strains were selected from a collection of $K$. pneumoniae isolates recovered from three tertiary hospitals in Southwest China (Guizhou Provincial People's Hospital [ $n=85]$, Sichuan Provincial People's Hospital [ $n=38]$ and Affiliated Hospital of Zunyi Medical University $[n=36]$ ) between August 1st, 2018 and December 31st, 2019. Strains from a single patient were excluded. All CRKP strains were characterized using an automatic microbial analysis system (Phoenixtm-100, BD, USA), followed by amplification of the RNA polymerase $\beta$ subunit gene $(r p o B)$ and evaluation with a modified Hodge test in accordance with Clinical and Laboratory Standards Institute (CLSI) guidelines [11]. K. pneumoniae ATCC700603 was used as the quality control strain.

\section{Study Population}

Inpatients with clinical symptoms and positive CRKP culture from sputa, urine, blood, ascites or wound secretions were evaluated for CRKP infection according to the Centers for Disease Control and Prevention (CDC) and National Healthcare Safety Network (NHSN) criteria [12]. Hospital-acquired CRE infection was defined as an infection that occurred after more than $48 \mathrm{~h}$ of hospitalization. A CRKP case was defined as the first clinical culture with carbapenem-nonsusceptible (imipenem, meropenem, or ertapenem) $K$. pneumoniae for each individual patient.

\section{Data Collection}

Medical records of all cases in this study were reviewed to collect clinical and patient data, including specimen source, demographic characteristics, underlying medical conditions, indwelling devices 30 days prior to culture, infection type(s), antimicrobial therapy, laboratory tests and clinical outcomes. Patients with clinically significant hospital-acquired infection or healthcare-associated infection due to CRKP were eligible for this study. Exclusion criteria were community-acquired infection, missing key data, isolates with colonization only, screening samples and subsequent episodes in an individual patient. 


\section{Microbiological Investigation}

The minimal inhibitory concentrations (MICs) of 12 antibiotics, including meropenem (Sumitomo Pharmaceuticals, Suzhou, China), imipenem (MSD, Hangzhou, China), cefoxitin, gentamycin, amikacin (National Institutes for Food and Drug Control, Beijing, China), levofloxacin (Yangzijiang, Jiangsu, China), azithromycin, ceftriaxone (Roche, Shanghai, China), ceftazidime, aztreonam, tigecycline, piperacillin/tazobactam, and cefoperazone/sulbactam (Pfizer, NY, USA), against CRKP were determined by agar and broth dilution methods according to CLSI guidelines [13]. The interpretive criteria for tigecycline were based on the breakpoints of the Food and Drug Administration (FDA).

The presence of carbapenemase genes ( $b / a_{\mathrm{NDM}}, b / a_{\mathrm{KPC}}, b / a_{\mathrm{IMP}}, b / a_{\mathrm{GES}}, b / a_{\mathrm{OXA}-48}$ and $\left.b / a_{\mathrm{VIM}}\right)$ described in previous studies was assessed by Polymerase Chain Reaction (PCR) as previously described $[14,15]$. Primers are shown in Table 1. All amplified PCR products were submitted for direct sequencing twice on both strands with an automated DNA sequencer (ABI 3730XL, Weiterstadt, Germany). Nucleotide sequences were analyzed using the online basic local alignment search tool (BLAST) and Clustal W programs (multiple sequences alignment, pairwise comparisons of sequences and dendrograms) against the CRKP carbapenemase gene (GenBank).

DNA for multi-locus sequence typing (MLST) analysis was prepared using a commercial bacterial DNA extraction kit (TIANamp Bacteria DNA Kit, China) according to the manufacturer's instructions. MLST was performed according to the protocol described on the Pasteur Institute MLST website (http://bigsdb.pasteur. fr/klebsiella/klebsiella.html) with amplification of seven housekeeping loci (gapA, infB, $m d h, p g i, p h o E, r p o B$ and tonB) [12]. 
Table 1

Sequence of primers for amplification of carbapenem enolase gene and annealing temperature

\begin{tabular}{|c|c|c|c|}
\hline $\begin{array}{l}\text { PCR } \\
\text { target }\end{array}$ & Primer sequence $\left(5^{\prime}-3^{\prime}\right)$ & $\begin{array}{l}\text { Annealing temperature } \\
\left({ }^{\circ} \mathrm{C}\right)\end{array}$ & Fragment length (bp) \\
\hline \multirow[t]{2}{*}{ KPC-2 } & F: GTTTGTTGATTGGCTAAAGG & 52 & 203 \\
\hline & R: TGTGCTTGTCATCCTTGTTA & & \\
\hline \multirow[t]{2}{*}{ IMP } & F: TGAGCAAGTTATCTGTATTC & 60 & 740 \\
\hline & R: TTAGTTGCTTGGTTTTGATG & & \\
\hline \multirow[t]{2}{*}{ NDM-1 } & F: CAGCACACTTCCTATCTC & 54 & 292 \\
\hline & R: CCGCAACCATCCCCTCTT & & \\
\hline \multirow[t]{2}{*}{ GES } & $\begin{array}{l}\text { F: } \\
\text { GTTTTTGCAATGTGCTCAACG }\end{array}$ & 55 & 371 \\
\hline & R: TGCCATAGCAATAGGCGTAG & & \\
\hline \multirow[t]{2}{*}{ OXA-48 } & F: GCGTGTATTAGCCTTATCGG & 60 & 783 \\
\hline & R: TTTTCCTGTTTGAGCACTTC & & \\
\hline \multirow[t]{2}{*}{ VIM } & F: GATGGTGTTTGGTCGCAT & 60 & 390 \\
\hline & R: CGAATGCGCAGCACCAG & & \\
\hline
\end{tabular}

\section{Statistical analysis}

Enumeration data were represented by the number of cases and percentage [n (\%)]. Measurement data, disordered counting data and rank classification counting data were analyzed by ANOVA, Fisher's exact test and rank-sum test, respectively. Significance was assessed at $p<0.05$. All analyses were performed using SPSS software, Version 21.0.

\section{Results}

\section{Clinical data analysis}

Of 159 CRKP strains, 59.1\% (94/159) were isolated from male patients and 41.9\% (65/159) from female patients. About one-half of patients $(80 / 159,50.3 \%)$ were at least 65 years old, and $77.4 \%(123 / 159)$ patients had two or more comorbidities (Table 2). 
Table 2

Basic characteristics of patients with CRKP infection

\begin{tabular}{|c|c|}
\hline Projects & Number of \\
\hline \multicolumn{2}{|l|}{ Gender } \\
\hline Male & $94(59.1)$ \\
\hline Female & $65(40.9)$ \\
\hline Age Median (Quartile spacing) (year) & $64(52-78)$ \\
\hline $18-49$ & $37(23.3)$ \\
\hline $50-64$ & $42(26.4)$ \\
\hline $65-79$ & $51(32.1)$ \\
\hline$₫ 80$ & $29(18.2)$ \\
\hline Basic disease & $155(97.5)$ \\
\hline Diabetes & $37(23.3)$ \\
\hline Heart disease ${ }^{a}$ & $65(40.9)$ \\
\hline hypertension & $70(44.0)$ \\
\hline Immunodeficiency ${ }^{b}$ & $9(5.7)$ \\
\hline Liver disease $^{c}$ & $42(26.4)$ \\
\hline Nervous system diseasesd & $89(56.0)$ \\
\hline Respiratory system disease $e^{e}$ & 145(91.2) \\
\hline Kidney disease ${ }^{f}$ & $70(44.0)$ \\
\hline Hormone use & $42(26.4)$ \\
\hline Immunosuppression use & 0 \\
\hline Tumor ${ }^{g}$ & $14(8.9)$ \\
\hline ICU & $107(67.3)$ \\
\hline Smoking & $42(26.4)$ \\
\hline No basic disease & $4(2.5)$ \\
\hline
\end{tabular}

${ }^{a}$ Heart disease: congestive heart failure, coronary heart disease, valve replacement and congenital heart disease. ${ }^{b}$ Immunodeficiency: splenectomy, chemotherapy and agranulocytosis.

Qiver disease: hepatitis, liver cirrhosis, liver abscess, abnormal liver function and fatty liver. 
${ }^{d}$ Nervous system diseases: apoplexy, transient ischemic attack, central paralysis and meningitis.

${ }^{e}$ Respiratory system disease: COPD, asthma, interstitial lung disease, pneumonia and respiratory failure.

${ }^{f}$ Respiratory failure: chronic kidney disease and azotemia.

${ }^{g}$ Tumor: respiratory, digestive tract, obstetrics and gynecology, blood and nervous system tumors.

Clinical characteristics and outcomes are summarized in Table 3. Of the studied patients, $96.8 \%$ (150/159) required the use of indwelling devices prior to culture collection. Multiple antibiotics were used by $98.1 \%(156 / 159)$ of patients. The majority of the patients demonstrated both an increased white blood cell count $(129 / 159,81.1 \%)$ and elevated levels of C-reactive protein $(144 / 159,90.6 \%)$. All patients showed increased levels of procalcitonin (159/159, 100\%). Mortality among these 159 patients was $18.9 \%(30 / 159)$.

\section{Detection Of Carbapenemases}

At least one carbapenemase was detected in each of these 159 CRKP strains, among which there were 129 strains $(129 / 159,81.1 \%)$ producing only KPC-2, 21 strains $(21 / 159,13.2 \%)$ only producing NDM-1, and 9 strains $(9 / 159,5.7 \%)$ producing both KPC-2 and NDM-1. Of the CRKP strains found to produce both KPC-2 and NDM-1, 66.7\% (6/9) were isolated from bloodstream infections. The bla $a_{\mathrm{GES}}, b / a_{\mathrm{IMP}}, b / a_{\mathrm{VIM}}$ and $b / a_{0 X A-48}$ genes were not detected in any of these 159 CRKP strains.

\section{Antimicrobial Susceptibility Test}

All CRKP strains were found to be multi-drug resistance (MDR), and all showed resistance to imipenem, meropenem, gentamicin, ceftazidime, cefoxitin, cefoperazone/sulbactam and aztreonam. The resistance rate to both azithromycin and ceftriaxone was $96.9 \%$ (154/159), followed by piperacillin/tazobactam (145/159, 91.2\%), levofloxacin (136/159, 85.5\%) and amikacin (129/159, 81.8\%). A total of nine CRKP strains producing both KPC-2 and NDM- 1 were resistant to all tested antibiotics (Table 4). These KPC- $2^{+}$ $\mathrm{NDM}-1^{+}$strains were also characterized by higher MICs of imipenem, meropenem, ceftazidime, cefoxitin, cefoperazone/sulbactam, aztreonam and piperacillin/tazobactam compared to those of strains that only produce either KPC-2 or NDM-1 $(P<0.05)$. 
Table 3

Clinical characteristics, laboratory examination and outcome of patients with CRKP infection

\begin{tabular}{|c|c|c|c|c|c|c|}
\hline Projects & $\begin{array}{l}\text { Sputa } \\
(\%) \\
(n=81)\end{array}$ & $\begin{array}{l}\text { Blood }(\%) \\
(n=33)\end{array}$ & $\begin{array}{l}\text { Urine (\%) } \\
(n=18)\end{array}$ & $\begin{array}{l}\text { Ascites (\%) } \\
(n=15)\end{array}$ & $\begin{array}{l}\text { Wound } \\
\text { secretions } \\
(\%)(n=12)\end{array}$ & $\begin{array}{l}\mathrm{P} \\
\text { value }\end{array}$ \\
\hline \multicolumn{7}{|l|}{ Invasive procedures } \\
\hline Happened & 78(96.3) & $33(100.0)$ & $15(83.3)^{\mathrm{ab}}$ & $12(80.0)^{a b}$ & $12(100.0)$ & 0.013 \\
\hline $\begin{array}{l}\text { Deep venous } \\
\text { catheterization }\end{array}$ & $69(85.2)$ & $30(90.9)$ & 15(83.3) & 12(80.0) & $12(100.0)$ & 0.526 \\
\hline $\begin{array}{l}\text { tracheal intubation / } \\
\text { tracheostomy }\end{array}$ & $57(70.4)$ & $27(81.8)$ & $9(50.0)$ & $6(40.0)^{\mathrm{ab}}$ & $12(100.0)^{\text {acd }}$ & 0.001 \\
\hline Urinary catheter & $75(92.6)$ & $33(100.0)$ & $15(83.3)^{b}$ & $9(60.0)^{\mathrm{ab}}$ & $12(100.0)^{d}$ & 0.001 \\
\hline Dialysis & $6(7.4)$ & $3(9.1)$ & $0(0.0)$ & $0(0.0)$ & $3(25.0)$ & 0.127 \\
\hline \multicolumn{7}{|l|}{ Antibiotic use } \\
\hline Monotherapy & $0(0.0)$ & $0(0.0)$ & $0(0.0)$ & $3(20.0)^{a b c}$ & $0(0.0)$ & 0.001 \\
\hline Polypharmacy & 74(91.4) & $33(100.0)$ & $17(94.4)$ & 12(80.0) & 12(100.0) & 0.100 \\
\hline $\begin{array}{l}\text { third and fourth } \\
\text { generation } \\
\text { cephalosporins }\end{array}$ & $60(74.1)$ & $27(81.8)$ & $9(50.0)^{\mathrm{ab}}$ & $15(100.0)^{a c}$ & $12(100.0)^{\mathrm{ac}}$ & 0.002 \\
\hline Carbapenem & $48(59.3)$ & $27(81.8)^{a}$ & $9(50.0)^{b}$ & $9(60.0)$ & $12(100.0)^{\mathrm{acd}}$ & 0.004 \\
\hline \multicolumn{7}{|l|}{ Laboratory tests } \\
\hline Leucocyte & & & & & & 0.040 \\
\hline$₫ 4000$ & 18(22.2) & $6(18.2)$ & $6(33.3)$ & $0(0.0)$ & $0(0.0)$ & \\
\hline$\otimes 10000$ & $63(77.8)$ & $27(81.8)$ & 12(66.7) & $15(100.0)$ & $12(100.0)$ & \\
\hline C-reactive protein & & & & & & 0.141 \\
\hline$<5 \mathrm{mg} / \mathrm{L}$ & $3(3.7)$ & $0(0.0)$ & $0(0.0)$ & $0(0.0)$ & $0(0.0)$ & \\
\hline $5-10 \mathrm{mg} / \mathrm{L}$ & $6(7.4)$ & $3(9.1)$ & $0(0.0)$ & $0(0.0)$ & $3(25.0)$ & \\
\hline
\end{tabular}

Note: ${ }^{\mathrm{a} C o m p a r e d ~ w i t h ~ t h e ~ s p u t u m ~ g r o u p, ~} \mathrm{P}<0.05$

${ }^{\mathrm{b}}$ Compared with the blood group, $\mathrm{P}<0.05$

cCompared with urine group, $\mathrm{P}<0.05$

${ }^{\mathrm{d}}$ Compared with ascites group, $\mathrm{P}<0.05$ 


\begin{tabular}{|c|c|c|c|c|c|c|}
\hline Projects & $\begin{array}{l}\text { Sputa } \\
(\%) \\
(n=81)\end{array}$ & $\begin{array}{l}\text { Blood (\%) } \\
(n=33)\end{array}$ & $\begin{array}{l}\text { Urine (\%) } \\
(n=18)\end{array}$ & $\begin{array}{l}\text { Ascites (\%) } \\
(n=15)\end{array}$ & $\begin{array}{l}\text { Wound } \\
\text { secretions } \\
(\%)(n=12)\end{array}$ & $\begin{array}{l}\mathrm{P} \\
\text { value }\end{array}$ \\
\hline$>10 \mathrm{mg} / \mathrm{L}$ & $72(88.9)$ & $30(90.9)$ & $18(100.0)$ & $15(100.0)$ & $9(75.0)$ & \\
\hline Procalcitonin & & & & & & 0.063 \\
\hline $0.05-2 \mathrm{ng} / \mathrm{ml}$ & $36(44.4)$ & $15(45.5)$ & $12(66.7)$ & $3(20.0)$ & $3(25.0)$ & \\
\hline$\bigotimes 2 \mathrm{ng} / \mathrm{ml}$ & $27(33.3)$ & $15(45.5)$ & $6(33.3)$ & $3(20.0)$ & $6(50.0)$ & \\
\hline Course and outcome & & & & & & $\begin{array}{l}< \\
0.001\end{array}$ \\
\hline Rehabilitation & $17(21.0)$ & $2(6.1)$ & $6(33.3)$ & $3(20.0)$ & $5(41.7)$ & \\
\hline $\begin{array}{l}\text { Transfer (including } \\
\text { improvement) }\end{array}$ & $57(70.4)$ & $9(27.3)$ & $12(66.7)$ & $12(80.0)$ & $6(50.0)$ & \\
\hline Death & $7(8.6)$ & $22(66.6)$ & $0(0.0)$ & $0(0.0)$ & $1(8.3)$ & \\
\hline \multicolumn{7}{|c|}{ Note: ${ }^{\mathrm{a} C o m p a r e d}$ with the sputum group, $\mathrm{P}<0.05$} \\
\hline \multicolumn{7}{|c|}{${ }^{b}$ Compared with the blood group, $P<0.05$} \\
\hline \multicolumn{7}{|c|}{${ }^{\mathrm{C}}$ Compared with urine group, $\mathrm{P}<0.05$} \\
\hline${ }^{\mathrm{d}}$ Compared with ascite & group, $\mathrm{P}<\mathrm{C}$ & & & & & \\
\hline
\end{tabular}


Table 4

Minimal inhibitory concentration of 159 strains of CRKP against 12 antibiotics $(\mu \mathrm{g} / \mathrm{mL})$

\begin{tabular}{|llll|}
\hline & $\begin{array}{l}\text { Strains with } \\
\text { KPC-2 } \\
(\mathbf{n = 1 2 9 )}\end{array}$ & $\begin{array}{c}\text { Strains with } \\
\text { NDM-1 } \\
(\mathbf{n = 1 2 9})\end{array}$ & $\begin{array}{c}\text { Strains with both KPC-2 and } \\
\text { NDM-1 } \\
(\mathbf{n}=9)\end{array}$ \\
\hline Imipenem & $32-512$ & $16-256$ & $>512^{\mathrm{ab}}$ \\
\hline Meropenem & $16-512$ & $16-256$ & $>512^{\mathrm{ab}}$ \\
\hline Gentamicin & $16-256$ & $16-256$ & $16-256$ \\
\hline Amikacin & $1-512^{\mathrm{a}}$ & $1-8$ & $64-512$ \\
\hline Levofloxacin & $0.25-512^{\mathrm{a}}$ & $0.25-2$ & $32-512$ \\
\hline Azithromycin & $16-512^{\mathrm{a}}$ & $4-16$ & $>512^{\mathrm{ab}}$ \\
\hline Ceftriaxone & $64-512^{\mathrm{a}}$ & $0.5-128$ & $>512^{\mathrm{ab}}$ \\
\hline Ceftazidime & $16-512$ & $16-512$ & $>512^{\mathrm{ab}}$ \\
\hline Cefoxitin & $32-512$ & $32-512$ & $>512^{\mathrm{ab}}$ \\
\hline Aztreonam & $32-256$ & $32-256$ & $>512^{\mathrm{ab}}$ \\
\hline $\begin{array}{l}\text { Piperacillin / } \\
\text { tazobactam }\end{array}$ & $16 / 4-512 / 4^{\mathrm{a}}$ & $64 / 4-512 / 4$ & $>512 / 4^{\mathrm{ab}}$ \\
\hline $\begin{array}{l}\text { Cefoperazone / } \\
\text { sulbactam }\end{array}$ & $64-512$ & $16-256$ & $>512^{\mathrm{ab}}$ \\
\hline aCompared with NDM-1-producing strains, $\mathrm{p}<0.05$. & \\
\hline b Compared with KPC-2-producing strains, $\mathrm{p}<0.05$ & \\
\hline
\end{tabular}

\section{MLST}

MLST results showed that the most prevalent sequence type (ST) was ST11 (102/159, 64.2\%), with a total of eight STs detected across the 159 CRKP strains. STs were significantly different between Guizhou Provincial People's Hospital and Affiliated Hospital of Zunyi Medical University $(P<0.05)$. ST244 strains were isolated only from Affiliated Hospital of Zunyi Medical University (Table 5). 
Table 5

Multilocus sequence typing of 159 CRKP strains

\begin{tabular}{|lllll|}
\hline & $\begin{array}{l}\text { Guizhou Provincial } \\
\text { People's Hospital (\%) } \\
(\mathbf{n}=\mathbf{8 5})\end{array}$ & $\begin{array}{l}\text { Sichuan Provincial } \\
\text { People's Hospital (\%) } \\
(\mathbf{n}=\mathbf{3 8})\end{array}$ & $\begin{array}{l}\text { Affiliated Hospital of Zunyi } \\
\text { Medical University (\%) } \\
(\mathbf{n}=36)\end{array}$ & $\begin{array}{l}\text { Total (\%) } \\
(\mathbf{n}=\mathbf{1 5 9})\end{array}$ \\
\hline ST11 & $64(75.3)$ & $23(60.5)$ & $15(41.7)$ & $102(64.2)$ \\
\hline ST2792 & $4(4.7)$ & $3(7.9)$ & $2(5.6)$ & $9(5.7)$ \\
\hline ST524 & $3(3.5)$ & $5(13.2)$ & $1(2.8)$ & $9(5.7)$ \\
\hline ST35 & $3(3.5)$ & $1(2.6)$ & $1(2.8)$ & $5(3.1)$ \\
\hline ST789 & $5(5.9)$ & $4(10.5)$ & $10(27.8)$ & $19(11.9)$ \\
\hline ST1066 & $2(2.4)$ & $1(2.6)$ & $2(5.6)$ & $5(3.1)$ \\
\hline ST29 & $4(4.7)$ & $1(2.6)$ & $1(2.8)$ & $4(3.8)$ \\
\hline ST244 & 0(0.0) & $4(0.0)$ & $4(11.1)$ & \\
\hline aCompared with Guizhou Provincial People's Hospital strains, $p<0.05$. & \\
\hline
\end{tabular}

\section{Discussion}

CRKP presents a particularly critical problem worldwide due to rapidly rising resistance rates and subsequent high mortality. Prior to this study, there was limited understanding of the molecular epidemiology of CRKP in Southwest China. In this study, 159 CRKP strains were isolated from clinical samples taken at three tertiary hospitals across Southwest China. This research serves as a powerful supplement to multicenter studies of CRKP epidemiology in China.

According to this study, in Southwest China, death by CRKP infection was primarily caused by bloodstream infections (66.6\%). Meta-analysis shows that the mortality rates of CRKP infections in North America, South America, Europe and Asia were 33.24\%, 46.71\%, 50.06\% and 44.82\%, respectively [16]. The mortality rate of bloodstream CRKP infection reaches $54.3 \%$, which is quite high [16]. In this study, most of the patients with bloodstream infections experienced invasive operations such as deep vein catheterization, tracheal intubation and mechanical ventilation before the collection of a positive culture. One study, which collected 100 CRKP strains from Shanghai, China, reports that mechanical ventilation could lead to a higher CRKP infection rate [17]. In our study, all patients with bloodstream infections experienced antimicrobial therapy before CRKP culture, $81.8 \%$ of which were treated with either third- or fourth-generation cephalosporins or carbapenem. Hospitalization and history of antibiotic use -especially of $\beta$-lactams and carbapenems -- are considered independent risk factors for CRE infection [18, 19]. More than half of CRKP strains taken from bloodstream samples in our study produce both KPC-2 and NDM-1, which we found to be associated with higher MICs for a panel of antibiotics. 
The distribution of carbapenemases has been shown to regionally different; this study shows that KPC-2 is most prevalent in Southwest China, while NDM is the dominant CRKP carbapenemase in some provinces of China $[7,10,20]$. In the United States, the most prevalent mechanism of carbapenem resistance among Enterobacteriaceae is production of KPC, even though the combination of extendedspectrum $\beta$-lactamase (ESBLs)/AmpC cephalosporinase and membrane permeability can also make Klebsiella pneumoniae resistant to carbapenems [21, 22]. Recent reports indicate that KPC-producing, gram-negative isolates are being identified throughout the United States, as well as in parts of Europe, Asia and South America. [23-25]At present, it is believed that transmission of colonies and specific elements from existing drug-resistant strains is the main route for the emergence of new CRKP, suggesting that future control strategies should focus on inhibition of this gene transmission from drugresistant strains [10].

In this study, resistance rates to gentamicin, amikacin and levofloxacin were found to be $100 \%, 81.1 \%$ and $85.5 \%$, respectively. These values are higher than those reported from both other provinces of China and other countries [26, 27]. A retrospective study in China showed that, in an outbreak of nosocomial neonatal CRKP infection, the MICs of CRKP strains producing KPC-2 are higher than those of strains producing NDM-1 [28]. As mentioned above, a majority of CRKP strains (86.8\%) analyzed in this study were found to produce KPC-2. In addition, more than half of the patients in this study were seriously ill in the ICU, were over 65 years old and had previously experienced multiple antibiotics. CRKP treatment is still absolutely difficult, as in vitro antibiotic-sensitivity assays do not fully reflect the response of CRKP to medicines in vivo. Nephrotoxicity is still an issue that requires attention in clinical practice for CRKP infections, particularly with the use of amikacin or polymyxin [29]. For patients with CRKP blood infections, it has been suggested that dual therapy based on high-dose carbapenem may bring clinical benefits [30]. The use of new antibiotics, such as ceftazidime-avibactam, may be associated with better clinical prognosis and survival [27].

As previously reported, the majority of KPC-producing $K$. pneumoniae isolates in China belong to a common sequence type, ST11 (26). In contrast, a majority of these isolates in the United States belong to the ST258 type (27). Most of the CRKP strains in Southwest China belong to ST11 (64.2\%), consistent with its national prevalence [10]. In our previous studies, we compared the age, sex, infection and antimicrobial use of 37 individual patients with either ST11 or non-ST11 CRKP infections [31]. Although there is no statistically significant difference between the two groups, CRKP sequence type still plays a key role in tracing strains at the genotype level, facilitating our understanding of global epidemiology.

To the best of our knowledge, this is the first report of the molecular epidemiology of CRKP infections in Southwest China. This study is limited by the fact that the infection rate of CRKP in admitted patients could not be fully acquired. However, it is clear that CRKP isolates in Southwest China are strongly drugresistant to multiple antibiotics. In particular, patients with CRKP bloodstream infections have poor prognoses and high mortality. Moving forward, there is an urgent need to strengthen control measures and improve prevention of CRKP infections across Southwest China. 


\section{Declarations}

\section{Ethics approval and consent to participate}

The study was reviewed and approved by the research ethics committee of Guizhou Provincial People's Hospital (No. 2015022).

\section{Consent for publication}

The study does not contain any individual personal data, therefore, consent for publication is not required

\section{Availability of data and materials}

Data sharing not applicable to this article as no datasets were generated or analysed during the current study.

\section{Competing interests}

The authors declare that they have no competing interests

\section{Funding}

This study was funded by National Natural Science Foundation of China (81560003) and High-level Innovative Talents Foundation of Guizhou Province (GZSYQCC[2016]005).

\section{Authors' contributions}

Xiaoping Hu performed the experiment and drafted the article. Guohang Yuan and Yaoyao Wu participated in acquisition of data and performed data analysis. Weijia Liu and Xiangyan Zhang interpreted the data. Lin Liu conceived the study and participated in analysis and draft of the study.

\section{Acknowledgements}

Not applicable

\section{References}

1. Chen L, Mathema B, Pitout JD, DeLeo FR, Kreiswirth BN. Epidemic Klebsiella pneumoniae ST258 is a hybrid strain. mBio. 2014;5(3):e01355-14. doi:10.1128/mBio.01355-14.

2. Mills JP, Talati NJ, Alby K, Han JH. The Epidemiology of Carbapenem-Resistant Klebsiella pneumoniae Colonization and Infection among Long-Term Acute Care Hospital Residents. Infect Control Hosp Epidemiol. 2016;37(1):55-60. doi:10.1017/ice.2015.254.

3. Vital signs. carbapenem-resistant Enterobacteriaceae. MMWR Morb Mortal Wkly Rep. 2013;62(9):165-70. 
4. Hirsch EB, Tam VH. Detection and treatment options for Klebsiella pneumoniae carbapenemases (KPCs): an emerging cause of multidrug-resistant infection. J Antimicrob Chemother. 2010;65(6):1119-25. doi:10.1093/jac/dkq108.

5. Borer A, Saidel-Odes L, Riesenberg K, Eskira S, Peled N, Nativ R, et al. Attributable mortality rate for carbapenem-resistant Klebsiella pneumoniae bacteremia. Infect Control Hosp Epidemiol. 2009;30(10):972-6. doi:10.1086/605922.

6. Wei ZQ, Du XX, Yu YS, Shen P, Chen YG, Li LJ. Plasmid-mediated KPC-2 in a Klebsiella pneumoniae isolate from China. Antimicrob Agents Chemother. 2007;51(2):763-5. doi:10.1128/aac.01053-06.

7. Zhang R, Chan EW, Zhou H, Chen S. Prevalence and genetic characteristics of carbapenem-resistant Enterobacteriaceae strains in China. Lancet Infect Dis. 2017;17(3):256-7. doi:10.1016/s14733099(17)30072-5.

8. Hu FP, Guo Y, Zhu DM, Wang F, Jiang XF, Xu YC, et al. Resistance trends among clinical isolates in China reported from CHINET surveillance of bacterial resistance, 2005-2014. Clin Microbiol Infect. 2016;22(Suppl 1):9-14 doi:20.

9. Xu A, Zheng B, Xu YC, Huang ZG, Zhong NS, Zhuo C. National epidemiology of carbapenem-resistant and extensively drug-resistant Gram-negative bacteria isolated from blood samples in China in 2013. Clin Microbiol Infect. 2016;22(Suppl 1):1-8. doi:10.1016/j.cmi.2015.09.015.

10. Zhang Y, Wang Q, Yin Y, Chen H, Jin L, Gu B, et al. Epidemiology of Carbapenem-Resistant Enterobacteriaceae Infections: Report from the China CRE Network. Antimicrob Agents Chemother. 2018;62(2) doi:10.1128/aac.01882-17.

11. Methods for Dilution Antimicrobial Susceptibility Tests for Bacteria That Grow Aerobically Wayne P. Methods for Dilution Antimicrobial Susceptibility Tests for Bacteria That Grow Aerobically. Approved standard 10th edition. Clinical and Laboratory Standards Institute, CLSI document M07A10; 2015.

12. Horan TC, Andrus M, Dudeck MA. CDC/NHSN surveillance definition of health care-associated infection and criteria for specific types of infections in the acute care setting. Am J Infect Control. 2008;36(5):309-32. doi:10.1016/j.ajic.2008.03.002.

13. Wayne PJC, Institute LS. Clinical and Laboratory Standards Institute: Performance standards for antimicrobial susceptibility testing: Twenty-sixth informational supplement, M100-S. 2016;34(1).

14. Wang X, Xu X, Li Z, Chen H, Wang Q, Yang P, et al. An outbreak of a nosocomial NDM-1-producing Klebsiella pneumoniae ST147 at a teaching hospital in mainland China. Microb Drug Resist. 2014;20(2):144-9. doi:10.1089/mdr.2013.0100.

15. Liu YY, Wang Y, Walsh TR, Yi LX, Zhang R, Spencer J, et al. Emergence of plasmid-mediated colistin resistance mechanism MCR-1 in animals and human beings in China: a microbiological and molecular biological study. Lancet Infect Dis. 2016;16(2):161-8. doi:10.1016/s1473-3099(15)004247.

16. Xu L, Sun X, Ma X. Systematic review and meta-analysis of mortality of patients infected with carbapenem-resistant Klebsiella pneumoniae. Ann Clin Microbiol Antimicrob. 2017;16(1):18. 
doi:10.1186/s12941-017-0191-3.

17. Zheng B, Dai Y, Liu Y, Shi W, Dai E, Han Y, et al. Molecular Epidemiology and Risk Factors of Carbapenem-Resistant Klebsiella pneumoniae Infections in Eastern China. Front Microbiol. 2017;8:1061. doi:10.3389/fmicb.2017.01061.

18. Patel N, Harrington S, Dihmess A, Woo B, Masoud R, Martis P, et al. Clinical epidemiology of carbapenem-intermediate or -resistant Enterobacteriaceae. J Antimicrob Chemother. 2011;66(7):1600-8. doi:10.1093/jac/dkr156.

19. Wang Q, Zhang Y, Yao X, Xian H, Liu Y, Li H, et al. Risk factors and clinical outcomes for carbapenemresistant Enterobacteriaceae nosocomial infections. Eur J Clin Microbiol Infect Dis. 2016;35(10):1679-89. doi:10.1007/s10096-016-2710-0.

20. Yan J, Pu S, Jia X, Xu X, Yang S, Shi J, et al. Multidrug Resistance Mechanisms of Carbapenem Resistant Klebsiella pneumoniae Strains Isolated in Chongqing, China. Ann Lab Med. 2017;37(5):398-407. doi:10.3343/alm.2017.37.5.398.

21. Goodman KE, Simner PJ, Tamma PD, Milstone AM. Infection control implications of heterogeneous resistance mechanisms in carbapenem-resistant Enterobacteriaceae (CRE). Expert Rev Anti Infect Ther. 2016;14(1):95-108. doi:10.1586/14787210.2016.1106940.

22. Tamma PD, Goodman KE, Harris AD, Tekle T, Roberts A, Taiwo A, et al. Comparing the Outcomes of Patients With Carbapenemase-Producing and Non-Carbapenemase-Producing CarbapenemResistant Enterobacteriaceae Bacteremia. Clin Infect Dis. 2017;64(3):257-64. doi:10.1093/cid/ciw741.

23. Kitchel B, Rasheed JK, Patel JB, Srinivasan A, Navon-Venezia S, Carmeli Y, et al. Molecular epidemiology of KPC-producing Klebsiella pneumoniae isolates in the United States: clonal expansion of multilocus sequence type 258. Antimicrob Agents Chemother. 2009;53(8):3365-70. doi:10.1128/aac.00126-09.

24. Patel JB, Rasheed JK, Kitchel BJCMN. Carbapenemases in Enterobacteriaceae: activity, epidemiology, and laboratory detection. 2009;31(8):55-62.

25. Queenan AM, Bush K. Carbapenemases: the versatile beta-lactamases. Clin Microbiol Rev. 2007;20(3):440-58. doi:10.1128/cmr.00001-07.

26. Han JH, Goldstein EJ, Wise J, Bilker WB, Tolomeo P, Lautenbach E. Epidemiology of CarbapenemResistant Klebsiella pneumoniae in a Network of Long-Term Acute Care Hospitals. Clin Infect Dis. 2017;64(7):839-44. doi:10.1093/cid/ciw856.

27. Dong F, Zhang Y, Yao K, Lu J, Guo L, Lyu S, et al. Epidemiology of Carbapenem-Resistant Klebsiella pneumoniae Bloodstream Infections in a Chinese Children's Hospital: Predominance of New Delhi Metallo- $\beta$-Lactamase-1. Microb Drug Resist. 2018;24(2):154-60. doi:10.1089/mdr.2017.0031.

28. Yu J, Tan K, Rong Z, Wang Y, Chen Z, Zhu X, et al. Nosocomial outbreak of KPC-2- and NDM-1producing Klebsiella pneumoniae in a neonatal ward: a retrospective study. BMC Infect Dis. 2016;16(1):563. doi:10.1186/s12879-016-1870-y. 
29. Shields RK, Nguyen MH, Chen L, Press EG, Potoski BA, Marini RV, et al. Ceftazidime-Avibactam Is Superior to Other Treatment Regimens against Carbapenem-Resistant Klebsiella pneumoniae Bacteremia. Antimicrob Agents Chemother. 2017;61(8) doi:10.1128/aac.00883-17.

30. Giannella M, Trecarichi EM, Giacobbe DR, De Rosa FG, Bassetti M, Bartoloni A, et al. Effect of combination therapy containing a high-dose carbapenem on mortality in patients with carbapenemresistant Klebsiella pneumoniae bloodstream infection. Int J Antimicrob Agents. 2018;51(2):244-8. doi:10.1016/j.ijantimicag.2017.08.019.

31. Yang X, Liu L, Zhao D, Zhang X. Detection of drug resistance gene of carbapenem-resistant Klebsiella pneumoniae and molecular epidemiological study. Chongqing Medicine. 2018;047(15):1977-85.

\section{Supplementary Files}

This is a list of supplementary files associated with this preprint. Click to download.

- Mainmanuscript.pdf 